\title{
Discovery of twelve ZZ Ceti stars
}

\author{
B. G. Castanheira*, S. O. Kepler ${ }^{\dagger}$, S. J. Kleinman**, A. Nitta** and L. \\ Fraga $^{\ddagger}$ \\ *Institut für Astronomie, Türkenschanzstr. 17, A-1180 Wien, Austria \\ ${ }^{\dagger}$ Departamento de Astronomia, Universidade Federal do Rio Grande do Sul, \\ Av. Bento Gonçalves 9500, Porto Alegre 91501-970, RS, Brazil \\ ${ }^{* *}$ Gemini Observatory, 670 North A'ohoku Place, Hilo, HI 96720, USA \\ ${ }^{*}$ Southern Observatory for Astrophysical Research, Casilla 603, La Serena, Chile
}

\begin{abstract}
Using the SOAR 4.1-m telescope, we discovered low amplitude pulsations in three stars previously reported as Not-Observed-to-Vary (NOV), which are inside the ZZ Ceti instability strip. We also report the variability of nine new pulsating stars, bringing the total number of known ZZ Ceti stars to 149. In addition, we lowered the detection limit for 10 NOVs located near the edges of the instability strip. Our results are consistent with a pure mass-dependent ZZ Ceti instability strip.
\end{abstract}

Keywords: Pulsations, oscillations, and stellar seismology

PACS: $97.10 . \mathrm{Sj}$

\section{INTRODUCTION}

The ZZ Ceti stars are pulsating white dwarfs with hydrogen-dominated atmosphere, observed in a narrow instability strip, between 10800 and $12300 \mathrm{~K}$ [e.g. [2, 14]], with a small dependency on mass [8].

Mukadam et al. [14] and Mullally et al. [17] reported twenty stars as Not-Observedto-Vary (NOV) in the instability strip. Gianninas et al. [6, 7] proposed that the instability strip is pure, based on their $100 \%$ success rate in predicting variability, if temperature and surface gravity determinations are derived from $\mathrm{S} / \mathrm{N}>60$ spectra. They studied a bright sample, from the catalog of McCook \& Sion [13]. Gianninas et al. [6, 7] claimed that the uncertainties in temperature and mass were large enough to scatter pulsators outside the instability strip and constant stars, inside. Kepler et al. [11] re-observed four stars with $T_{\text {eff }} \sim 12000 \mathrm{~K}$ from the SDSS sample with GMOS at the Gemini $8 \mathrm{~m}$ telescope. Fitting these $S / N \geq 60$ spectra, they estimated the real uncertainties in the fits of Kleinman et al. [12] and Eisenstein et al. [5] are larger by $60 \%$ in temperature and a factor of 4 in $\log g$. However, the main component of their disagreement was systematic, with an average difference from the SDSS catalog measurements $[12,5]$ in temperature of $320 \mathrm{~K}$, systematically lower, and in $\log g$ of 0.24 dex, systematically larger. It would appear that low S/N of the SDSS spectra are not the main explanation for the possibly contaminated instability strip. There should be also some scatter of pulsators out of the strip and constant stars inside.

We continue the effort to determine whether the ZZ Ceti instability strip is a normal evolutionary stage in the white dwarf evolution or not. We report the variability of three ZZ Ceti stars previously classified as NOVs and nine previously unobserved variables. In our searches, we also lowered the detection limit for ten NOVs near the edges of

\footnotetext{
CP1273, $17^{\text {th }}$ European White Dwarf Workshop edited by M. K. Werner and T. Rauch

(c) 2010 American Institute of Physics 978-0-7354-0823-4/10/\$30.00
} 
the strip. Since we have discovered low amplitude pulsations in every NOV we have observed, our observational evidence is for a pure ZZ Ceti instability strip. However, we will only be able to claim that the ZZ Ceti instability strip is truly pure when we lower the detection limits for variability of all stars within the boundaries and on the edges of the strip and have more accurate $T_{\text {eff }}$ and $\log g$ determinations for all stars near the edges and inside the instability strip.

\section{OBSERVATIONS AND DATA REDUCTION}

We are looking for pulsators among the white dwarfs discovered with the SDSS [1]. Kleinman et al. [12] describes the fitting process for all SDSS white dwarfs. We chose to observe DAs with previous NOV limits higher than $1 \mathrm{mma}$ [3]. along with previously un-observed DAs within the observed instability strip.

We observed with the $4.1 \mathrm{~m}$ SOAR telescope, in Chile, using the SOAR Optical Imager, a mosaic of two EEV $2048 \times 4096$ CCDs, thinned and back illuminated, with an efficiency around $73 \%$ at $4000 \AA$, at the Naysmith focus. The integration times were $30 \mathrm{~s}$. We used fast readout mode with the CCDs binned $4 \times 4$ to decrease the readout+write time to $6.4 \mathrm{~s}$ and still achieve $0.354 " /$ pixel resolution. The Bessel $B$ filter was used to maximize the amplitude and minimize the red fringing.

We reduced the data using $h s p$ (high speed photometry) scripts, developed by Antonio Kanaan for IRAF, with weighted apertures, for time-series photometry [9]. We extracted light curves of all bright stars observed simultaneously in the field. Then, we divided the light curve of the target star by the sum of the comparison stars to minimize effects of sky and transparency fluctuations. We chose the aperture size by optimizing the noise in the resulting Fourier transform.

As an objective criterion, we determined a power amplitude limit such that a peak exceeding this limit has a $1 / 1000$ probability of being due to noise (false alarm probability or FAP) $[18,10]$ For each light curve, we calculated the ratio $P_{0} /\langle P\rangle=\ln \left(\frac{1}{1000 * N}\right)$, where $P_{0}$ is the power amplitude of a peak, $\langle P\rangle$ is the average in the power spectrum, and $N$ is the number of independent samples.

We observed most targets at two separate times, each for about two hours, to look for coherent signals in the light curves. We also checked if smaller peaks in the Fourier transform were intrinsic variations of the star. We subtracted from the original light curve the sinusoid representing the highest amplitude peak, re-calculated the Fourier transform and the new noise level, and continued pre-whitening until the highest remaining peak has $\mathrm{FAP}>1 / 1000$.

\section{NEW ZZ CETI STARS}

In Table 1, we list the properties of the new ZZ Ceti stars.

The star SDSS J220915.84-091942.5 is a typical red edge pulsator, the cooler end of the instability strip, with high amplitude and long periodicities. For this star, we detected two independent periodicities, as well as the first harmonic of the main mode at $\sim 895 \mathrm{~s}$. SDSS J092511.60+050932.4 also pulsates with long periods, but with small amplitude. 
TABLE 1. Observational properties of the new ZZ Ceti stars. $T_{\text {eff }}$ and $\log g$ from SDSS spectra.

\begin{tabular}{ccccccc}
\hline Star (SDSS J) & $T_{\text {eff }}(\mathrm{K})$ & $\log g$ & Mass $\left(M_{\odot}\right)$ & $\mathrm{g}(\mathrm{mag})$ & Period (s) & $\mathrm{A}(\mathrm{mma})$ \\
\hline $004345.78+005549.9$ & $11820 \pm 190$ & $7.94 \pm 0.10$ & $0.58 \pm 0.05$ & 18.74 & 258.24 & 6.69 \\
\hline $012234.68+003025.8$ & $11800 \pm 50$ & $7.87 \pm 0.02$ & $0.54 \pm 0.01$ & 17.29 & 121.07 & 1.53 \\
& & & & & 200.75 & 1.25 \\
\hline $012950.44-101842.0$ & $11910 \pm 130$ & $8.00 \pm 0.03$ & $0.61 \pm 0.02$ & 18.32 & 193.76 & 2.88 \\
& & & & & 147.42 & 2.33 \\
\hline $030153.81+054020.0$ & $11470 \pm 50$ & $8.09 \pm 0.03$ & $0.66 \pm 0.02$ & 18.05 & 300.83 & 24.87 \\
\hline $092511.60+050932.4$ & $10880 \pm 30$ & $8.41 \pm 0.02$ & $0.87 \pm 0.01$ & 15.20 & 1127.14 & 3.17 \\
& & & & & 1264.29 & 3.05 \\
\hline $095936.96+023828.4$ & $11840 \pm 110$ & $8.05 \pm 0.06$ & $0.64 \pm 0.04$ & 18.15 & 283.41 & 12.95 \\
& & & & & 194.68 & 7.23 \\
\hline $110525.70-161328.5$ & $11670 \pm 90$ & $8.23 \pm 0.03$ & $0.75 \pm 0.02$ & 17.54 & 192.66 & 12.09 \\
& & & & & 298.25 & 7.09 \\
\hline $113604.01-013658.1$ & $11710 \pm 70$ & $7.96 \pm 0.04$ & $0.59 \pm 0.02$ & 17.84 & 260.79 & 2.45 \\
\hline $133831.74-002328.0$ & $11870 \pm 80$ & $8.13 \pm 0.04$ & $0.69 \pm 0.02$ & 17.09 & 196.93 & 3.97 \\
& & & & & 119.72 & 1.75 \\
\hline $214723.73-001358.4$ & $11990 \pm 290$ & $7.92 \pm 0.11$ & $0.57 \pm 0.06$ & 18.98 & 199.77 & 3.88 \\
\hline $220915.84-091942.5$ & $11430 \pm 110$ & $8.33 \pm 0.06$ & $0.82 \pm 0.04$ & 18.93 & 894.71 & 43.94 \\
& & & & & 447.94 & 10.80 \\
& & & & & 789.31 & 10.37 \\
\hline $233726.28-010110.9$ & $11380 \pm 190$ & $7.90 \pm 0.12$ & $0.56 \pm 0.06$ & 18.85 & 494.66 & 40.07 \\
& & & & & 725.49 & 26.09 \\
& & & & & 304.17 & 11.71 \\
\hline
\end{tabular}

This star could be an example of a ZZ Ceti on the verge of leaving the instability strip Mukadam et al. [16], Castanheira \& Kepler [4]. The other new pulsators are closer to the blue edge of the instability strip, with low amplitude and short periods.

The stars SDSS J012234.68+003025.8, SDSS J113604.01-013658.1, and SDSS J133831.74-002328.0 were previously reported as NOV2, NOV2, and NOV4 Mukadam et al. [14] and Mullally et al. [17]. Our observations achieved a lower noise level, revealing that these stars are low amplitude pulsators, pulsating with short periods, typical of blue edge stars, consistent with our temperature determinations.

\section{NEW ZZ CETI INSTABILITY STRIP}

In the Figure 1, we plot the ZZ Ceti instability strip. There are still more than a dozen NOVs inside the current observed instability strip. The question whether all the remaining NOVs pulsate with amplitudes below the published detection limits remains, since all new low amplitude pulsators have amplitudes smaller than the previous $4 \mathrm{mma}$ average limit. Our observations point towards a pure instability strip, but there is no guaran- 


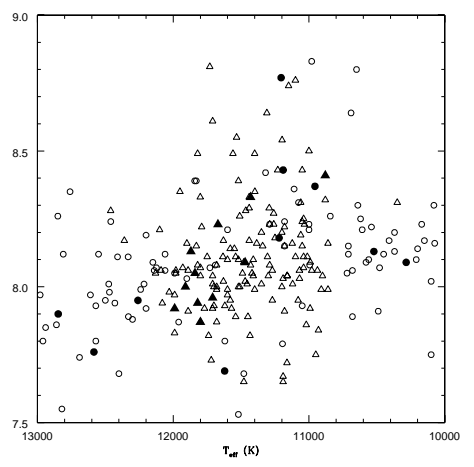

FIGURE 1: New ZZ Ceti instability strip. The full triangles are the ZZ Ceti stars we discovered, the open triangles are the previously known ZZ Ceti stars, the full circles are the NOVs for which we lowered the detection limits, and the open circles are the NOVs [14, $17,6] . T_{\text {eff }}$ and $\log g$ are from the spectroscopic determinations.

tee that other physical mechanisms cannot shut down pulsations. Therefore, we encourage the search of variability for the stars inside and at the edges of the instability strip previously reported as NOVs, reaching a detection limit of $\sim 1 \mathrm{mma}$, before declaring them non-pulsators.

\section{ACKNOWLEDGMENTS}

We acknowledge support from the CNPq-Brazil and the Stars research group at UT Austin and Österreichische Forschungsgemeinschaft for travel support.

\section{REFERENCES}

1. Abazajian, K. N., et al. 2009, ApJS, 182, 543

2. Bergeron, P., Fontaine, G., Billères, M., Boudreault, S., \& Green, E. M. 2004, ApJ, 600, 404

3. Castanheira, B. G., et al. 2007, A\&A, 462, 989

4. Castanheira, B. G., \& Kepler, S. O. 2009, MNRAS, 396, 1709

5. Eisenstein, D. J., et al. 2006, ApJS, 167, 40

6. Gianninas, A., Bergeron, P., \& Fontaine, G. 2005, ApJ, 631, 1100

7. Gianninas, A., Bergeron, P., \& Fontaine, G. 2006, AJ, 132, 831

8. Giovannini, O., Kepler, S. O., Kanaan, A., Wood, A., Claver, C. F., \& Koester, D. 1998, Baltic Astronomy, 7, 131

9. Kanaan, A., et al. 2005, A\&A, 432, 219

10. Kepler, S. O. 1993, Baltic Astronomy, 2, 515

11. Kepler, S. O., Castanheira, B. G., Costa, A. F. M., \& Koester, D. 2006, MNRAS, 372, 1799

12. Kleinman, S. J., et al. 2004, ApJ, 607, 426

13. McCook, G. P., \& Sion, E. M. 1999, ApJS, 121, 1

14. Mukadam, A. S., et al. 2004a, ApJ, 607, 982

15. Mukadam, A. S., Winget, D. E., von Hippel, T., Montgomery, M. H., Kepler, S. O., \& Costa, A. F. M. $2004 \mathrm{~b}, \mathrm{ApJ}, 612,1052$

16. Mukadam, A. S., Montgomery, M. H., Winget, D. E., Kepler, S. O., \& Clemens, J. C. 2006, ApJ, 640, 956

17. Mullally, F., Thompson, S. E., Castanheira, B. G., Winget, D. E., Kepler, S. O., Eisenstein, D. J., Kleinman, S. J., \& Nitta, A. 2005, ApJ, 625, 966

18. Scargle, J. D. 1982, ApJ, 263, 835 
Copyright of AIP Conference Proceedings is the property of American Institute of Physics and its content may not be copied or emailed to multiple sites or posted to a listserv without the copyright holder's express written permission. However, users may print, download, or email articles for individual use. 